\title{
Outcomes of resection for hepatocellular carcinoma with macroscopic bile duct tumour thrombus: A propensity score matched study
}

\author{
QIYU CHI ${ }^{1}$, ZHENG SHI $^{1}$, ZHIBO ZHANG $^{1}$, XIANG ZHANG $^{1}$, LI ZHANG $^{2}$ and SHANGENG WENG ${ }^{1}$ \\ Departments of ${ }^{1}$ Hepatopancreatobiliary Surgery and ${ }^{2}$ Pathology, \\ The First Affiliated Hospital of Fujian Medical University, Fuzhou, Fujian 350005, P.R. China
}

Received February 6, 2020; Accepted July 13, 2020

DOI: $10.3892 / \mathrm{ol} .2020 .11979$

\begin{abstract}
The incidence of hepatocellular carcinoma (HCC) with bile duct tumour thrombus (BDTT) is low, and related studies, especially studies on long-term survival, are uncommon. The present study aimed to evaluate the clinicopathological characteristics, prognostic factors and postoperative long-term outcomes of BDTT in patients with HCC. The clinicopathological characteristics and postoperative long-term outcomes of patients with HCC both with and without BDTT were compared before and after propensity score matching (PSM). Prognostic risk factors were assessed by Cox proportional hazards regression analyses after PSM. Tumour stages in the BDTT group were significantly higher than those in the group without BDTT $(\mathrm{P}=0.001)$. Overall survival (OS) and recurrence-free survival (RFS) rates were significantly higher in the group without BDTT than in the BDTT group before $\mathrm{PSM}(\mathrm{P}<0.001$ and $\mathrm{P}=0.003$, respectively). However, no significant difference in OS or RFS was found between the two groups after PSM $(\mathrm{P}=0.249$ and $\mathrm{P}=0.121$, respectively). Moreover, the median OS and RFS times of the BDTT patients who underwent tumour thrombectomy and bile duct resection were not significantly different $(\mathrm{P}=0.891$ and $\mathrm{P}=0.787$, respectively). In the multivariate analysis, macrovascular invasion (HR, 3.701; 95\% CI, 1.313-9.10.437; $\mathrm{P}=0.013$ ) was the only independent predictor of OS. Although the clinicopathological characteristics of the BDTT group suggested more advanced stage disease and poorer oncological outcomes than the group without BDTT, BDTT was not a poor prognostic factor for patients with $\mathrm{HCC}$ who underwent liver resection. Curative resection is recommended for patients with HCC and BDTT,
\end{abstract}

Correspondence to: Professor Shangeng Weng, Department of Hepatopancreatobiliary Surgery, The First Affiliated Hospital of Fujian Medical University, 20 Chazhong Road, Fuzhou, Fujian 350005, P.R. China

E-mail: shangeng@sina.com

Key words: hepatocellular carcinoma, bile duct tumour thrombus, liver resection, propensity score matching, long-term outcomes even for those with poor liver function, after proper perioperative management in order to achieve good long-term survival.

\section{Introduction}

Hepatocellular carcinoma (HCC) is the fifth most common malignancy, accounting for 600,000-700,000 deaths worldwide in 2014, and its incidence is increasing in Western countries $(1,2)$. HCC often invades the portal vein and hepatic vein or forms a venous tumour thrombus in the inferior vena cava. However, HCC with bile duct tumour thrombus (BDTT) is relatively rare and has been identified in $0.99-13 \%$ of autopsy and surgical specimens (2-6). Once BDTT in HCC is considered as advanced liver cancer, palliative treatment is often performed due to a misunderstanding of the cause of the obstructive jaundice, cholestasis and hepatic dysfunction (7-9). The complexity of the disease causes considerable challenges for clinical diagnosis and treatment. In recent years, patients with BDTT have more often been treated with surgery in a number of medical centres due to advances in diagnosis, surgical techniques and perioperative management $(4,10)$, and the outcomes of surgical treatment have been widely accepted to be significantly improved compared with those of palliative treatment $(11,12)$. However, due to the rarity of the disease, the clinicopathological characteristics are not well recognized and a limited number of studies are available, especially studies regarding long-term postoperative outcomes when compared with those of patients without BDTT; the possibility of selection bias therefore remains. Furthermore, whether extrahepatic bile duct resection is necessary remains controversial.

The present study aimed to evaluate the clinicopathological features and long-term oncological outcomes of patients with HCC and macroscopic BDTT. To overcome selection bias, propensity score matching (PSM) was applied and prognostic factors after liver resection were analysed.

\section{Patients and methods}

Patients. Between January 2013 and November 2018, 773 patients with HCC underwent hepatectomy at the Department of Hepatopancreatobiliary Surgery, The First Affiliated Hospital of Fujian Medical University (Fuzhou, 
China). Patients who had previously received locoregional therapy, such as hepatectomy ( $\mathrm{n}=63)$, transarterial chemoembolization (TACE; $n=11$ ), radiofrequency ablation (RFA; $\mathrm{n}=4)$, Gamma Knife ${ }^{\circledR}$ treatment ( $\left.\mathrm{n}=1\right)$ or TACE combined with RFA $(n=4)$, were excluded. Patients were also excluded if the tumour had been found to rupture $(n=25)$, invade peripheral organs or metastasize $(n=2)$ during the surgery. Patients whose data were incomplete or those who were lost to follow-up $(n=32)$ were also excluded from this study. Ultimately, a total of 631 patients (age range, 20-85 years) were included in the current study, 25 (3.96\%) of whom were allocated to the HCC with macroscopic BDTT group, and 606 (96.04\%) of whom were allocated to the group without BDTT. The clinicopathological characteristics and survival rates of the two groups were compared. To decrease possible confounding and selection biases of the baseline characteristics in the two groups, 1:1 matching variables [age, sex, body mass index, presence of comorbidity, $\alpha$-fetoprotein (AFP), hepatitis B virus, hepatitis C virus, indocyanine green retention rate at $15 \mathrm{~min}$, Child-Pugh classification (13), maximum tumour size, number of tumours, cirrhosis, macrovascular invasion, microvascular invasion, tumour differentiation, American Joint Committee on Cancer (AJCC) stage (14) and resection margins] were applied using PSM. As a result, 50 patients were divided into two groups: The BDTT group $(\mathrm{n}=25)$ and the group without BDTT $(\mathrm{n}=25)$. The clinicopathological characteristics and survival rates of the two groups were compared once more after PSM and the prognostic risk factors of HCC patients who underwent liver resection were assessed by the Cox proportional hazards model after excluding other influencing factors. In addition, the curative effects on BDTT in patients with HCC who underwent tumour thrombectomy and bile duct resection were compared. The current study was approved by the Institutional Review Board of The First Affiliated Hospital of Fujian Medical University and performed in accordance with the ethical guidelines of the institute.

Preoperative evaluation and management. Primary tumours were evaluated by contrast-enhanced thin-slice computed tomography (CT) or magnetic resonance imaging (MRI) and MR cholangiopancreatography. Liver function and whether another comorbidity was present were also assessed. Liver function was evaluated using the Child-Pugh classification system. An attempt was made to convert Child-Pugh class B disease into class A disease via appropriate preoperative treatment. To improve liver function and treat cholangitis, it was necessary to perform biliary drainage by percutaneous transhepatic biliary drainage (PTBD) or to use an endoscopic retrograde cholangiopancreatography in certain patients. The serum total bilirubin (TBIL) level of patients with obstructive jaundice after appropriate preoperative management needed to decrease to $<2.0 \mathrm{mg} / \mathrm{dl}$ or by $>50 \%$ before major hepatectomy was performed (normal range, $0.1-1.0 \mathrm{mg} / \mathrm{dl}$ ).

Surgical strategy. The surgical strategy was determined preoperatively at the department specialist meeting (similar to a multidisciplinary team conference). Sometimes, adjustments were necessary during surgery to select a reasonable procedure based on intraoperative exploration. The basic policy for the surgical treatment of HCC in The First Affiliated Hospital of
Fujian Medical University is that if the remaining liver function is sufficient, there is an inclination to perform an anatomical resection; non-anatomical resection is recommended only when the tumour is small, the position is superficial or the remaining liver function is insufficient. Anatomical resection involved resection of the tumour with its related portal vein branches and corresponding hepatic region. The surgical treatment strategy was complete resection of the primary HCC and BDTT, trying not to remove the extrahepatic bile duct, similar to the 'peeling off technique' (10). The extrahepatic bile duct was resected only when the BDTT could not be completely peeled off or would have violated the bile duct wall.

Pathological evaluation. After the specimens were stored and fixed in $10 \%$ formalin at room temperature for $12 \mathrm{~h}$, they were embedded in paraffin and cut into 5- $\mu \mathrm{m}$-thick sections. Hematoxylin and eosin staining (performed for $10 \mathrm{~min}$ at room temperature) and a light microscope (Nikon Corporation; magnification, $\mathrm{x} 100, \mathrm{x} 200$ and $\mathrm{x} 400$; analyzed using QImaging MicroPublisher 3.3 with Real-Time Viewing; Teledyne QImaging) were used. The numbers, sizes (maximum tumour diameter) and locations of the tumours were recorded during a macroscopic examination of the resected specimens by a pathologist. Moreover, the microscopic examination included determination of the histological differentiation, invasion of the bile duct (the size and location of BDTT), microvasculature and macrovasculature, the specimen margin and the presence of cirrhosis. The histological differentiation of HCC was assigned according to the Edmondson-Steiner system (15). Tumour stage was classified in accordance with the criteria of the AJCC (8th edition). HCC with macroscopic BDTT represents a tumour thrombus found in the common hepatic duct or the first to second branches of the intrahepatic bile duct. Classification of HCC with macroscopic BDTT was in accordance with the criteria of the Ueda classification (3).

Follow-up. Specially trained researchers used outpatient records and telephone calls to follow-up with the patients. After discharge from the hospital, AFP tests, ultrasound (US) and contrast-enhanced CT or MRI were performed at least every 3 months during the follow-up period. If recurrence or metastasis was observed, follow-up treatments, such as TACE, RFA, microwave ablation, reoperation and targeted drug administration, such as Sorafenib ( $400 \mathrm{mg}$ bid), Rivatinib (8-12 mg qd) or Regorafenib (160 mg qd), were performed, as determined by the patient's condition. Survival time was calculated from the date of surgery to the date of last contact, death or collection of survival information. The follow-up deadline was May 2019.

Statistical analysis. PSM was performed with a multiple factor logistic regression model, and a calliper of 0.10 of the standard deviation of the logit was imposed. Continuous variables are reported as the mean \pm standard deviation, and categorical variables are reported as the count and percentage. Continuous variables were compared using Student's t-test or the Mann-Whitney U test as appropriate. Categorical variables were compared using the $\chi^{2}$ test or Fisher's exact test if necessary. Patient survival rates were analysed by the Kaplan-Meier method, and survival curves were compared between groups using the log-rank test. 
Table I. Clinical features and operative procedures of patients with macroscopic BDTT.

\begin{tabular}{|c|c|c|c|c|c|c|c|c|}
\hline $\begin{array}{l}\text { Patient } \\
\text { no. }\end{array}$ & Sex & $\begin{array}{l}\text { Age, } \\
\text { years }\end{array}$ & $\begin{array}{l}\text { Location of } \\
\text { tip of BDTT }\end{array}$ & $\begin{array}{l}\text { Ueda } \\
\text { type }^{\mathrm{a}}\end{array}$ & $\begin{array}{c}\text { Biliary } \\
\text { decompression }\end{array}$ & $\begin{array}{l}\text { Operative } \\
\text { procedure }\end{array}$ & $\begin{array}{l}\text { Bile duct } \\
\text { resection }\end{array}$ & $\begin{array}{l}\text { Tumour } \\
\text { thrombectomy }\end{array}$ \\
\hline 1 & M & 54 & Left hepatic duct & 2 & No & Left hepatectomy & No & No \\
\hline 2 & M & 50 & CBD & 3 & No & Right hepatectomy & No & Yes \\
\hline 3 & M & 67 & CBD & 3 & Yes & Expand right hepatectomy & Yes & No \\
\hline 4 & M & 60 & CBD & 3 & Yes & Right hepatectomy & No & Yes \\
\hline 5 & $\mathrm{~F}$ & 61 & Right hepatic duct & 2 & No & Right hepatectomy & No & No \\
\hline 6 & M & 55 & CBD & 3 & Yes & Central hepatectomy & No & Yes \\
\hline 7 & $\mathrm{~F}$ & 64 & CBD & 3 & No & Right hepatectomy & No & Yes \\
\hline 8 & M & 65 & CBD & 3 & Yes & Right hepatectomy & No & Yes \\
\hline 9 & M & 39 & CBD & 3 & No & Right hepatectomy & Yes & No \\
\hline 10 & M & 65 & Right hepatic duct & 2 & No & Right hepatectomy & No & No \\
\hline 11 & M & 68 & CBD & 3 & No & Right hepatectomy & No & Yes \\
\hline 12 & $\mathrm{~F}$ & 49 & Left hepatic duct & 2 & No & Left hepatectomy & No & No \\
\hline 13 & M & 41 & CBD & 3 & Yes & Right hepatectomy & Yes & No \\
\hline 14 & M & 61 & CBD & 3 & Yes & Central hepatectomy & No & Yes \\
\hline 15 & M & 64 & CBD & 3 & No & Left hepatectomy & No & Yes \\
\hline 16 & M & 59 & CBD & 3 & No & Left hepatectomy & No & Yes \\
\hline 17 & M & 62 & CBD & 3 & Yes & Left hepatectomy & No & Yes \\
\hline 18 & $\mathrm{~F}$ & 44 & The second branch & 1 & No & Right posterior section & No & No \\
\hline 19 & $\mathrm{~F}$ & 70 & The second branch & 1 & No & Right posterior section & No & No \\
\hline 20 & M & 58 & CBD & 3 & No & Central hepatectomy & No & Yes \\
\hline 21 & M & 62 & Right hepatic duct & 2 & No & Right hepatectomy & No & No \\
\hline 22 & M & 55 & Left hepatic duct & 2 & No & Left hepatectomy & No & No \\
\hline 23 & $\mathrm{~F}$ & 63 & Left hepatic duct & 2 & No & Left hepatectomy & No & No \\
\hline 24 & M & 47 & Left hepatic duct & 2 & No & Left hepatectomy & No & No \\
\hline 25 & M & 44 & $\mathrm{CBD}$ & 3 & Yes & Left hepatectomy & Yes & No \\
\hline
\end{tabular}

${ }^{a}$ Type $3 \mathrm{a}$ or $3 \mathrm{~b}$ were classified together as type 3 . BDTT, bile duct tumour thrombus; CBD, common bile duct; M, male; F, female.

Patients who succumbed within 30 days of surgery were excluded from the survival analysis. Moreover, patients who underwent non-curative surgical resection were excluded from the recurrence analysis. Univariate and multivariate analyses were performed using Cox proportional hazards regression analyses. The variables found to have prognostic significance in the univariate analysis were entered into the Cox multivariate proportional hazards regression analysis to identify factors that independently predicted the HCC prognosis. $\mathrm{P}<0.05$ was considered to indicate a statistically significant difference. SPSS statistics software (version 24.0; IBM Corp.) was used for all statistical analyses.

\section{Results}

Perioperative outcomes of HCC patients with BDTT. The clinical features of the $25 \mathrm{HCC}$ patients with macroscopic BDTT are shown in Table I. The common bile duct (CBD) was the most frequent location of BDTT in this study (15 patients; $60 \%$ ), followed by the left hepatic duct (5 patients; $20 \%$ ), the right hepatic duct (3 patients; $12 \%$ ) and the second-order branch of the intrahepatic bile duct (2 patients; $8 \%$ ). According to the Ueda classification, BDTT was classified as type
1 in 2 patients (8\%), type 2 in 8 patients (32\%) and type 3 in 15 patients $(60 \%)$. No type 4 cases were included in this study. Among these patients, 11 (44\%) were diagnosed with obstructive jaundice on admission. A subsequent biliary drainage procedure was performed using PTBD guided by US in 8 patients (32\%), and 2 patients (8\%) underwent PTBD of the right hepatic duct and then PTBD of the left hepatic duct again on postoperative days 19 and 27. Another 3 patients $(12 \%)$ did not have biliary drainage as their TBIL increased in the short term and did not increase markedly before surgery. The median duration from biliary drainage to surgical treatment was 23 days (range, 13-42 days). A total of 17 patients $(68 \%)$ had TBIL levels $<2.0 \mathrm{mg} / \mathrm{dl}$ at the time of surgery, and TBIL levels decreased by $14.3 \pm 6.4 \mathrm{mg} / \mathrm{dl}$ at the time of preoperative biliary drainage. However, preoperative TBIL in the BDTT group was significantly higher than that in the without BDTT group $(1.98 \pm 1.72$ vs. $1.00 \pm 0.62 \mathrm{mg} / \mathrm{dl} ; \mathrm{P}=0.010$; data not shown).

The operative procedures used in the BDTT group are shown in Table I. The primary tumours were removed by right hemihepatectomy in 11 patients (44\%), including an extended right hemihepatectomy in 1 patient (4\%), by left hemihepatectomy in 9 patients (36\%), by central hepatectomy in 3 patients 
Table II. Clinicopathological characteristics before and after propensity score matching.

\begin{tabular}{|c|c|c|c|c|c|c|}
\hline \multirow[b]{2}{*}{ Variables } & \multicolumn{3}{|c|}{ All patients } & \multicolumn{3}{|c|}{ Propensity-matched patients } \\
\hline & $\begin{array}{l}\text { With BDTT } \\
\quad(\mathrm{n}=25)\end{array}$ & $\begin{array}{c}\text { Without } \\
\text { BDTT }(n=606)\end{array}$ & P-value & $\begin{array}{l}\text { With BDTT } \\
\quad(\mathrm{n}=25)\end{array}$ & $\begin{array}{c}\text { Without } \\
\text { BDTT }(\mathrm{n}=25)\end{array}$ & P-value \\
\hline Age, years & $57.08 \pm 8.98$ & $55 \pm 11.65$ & 0.290 & $57.08 \pm 8.98$ & $55.24 \pm 9.34$ & 0.479 \\
\hline Sex, n & & & 0.164 & & & $0.269^{\mathrm{a}}$ \\
\hline Male & 19 & 521 & & 19 & 22 & \\
\hline Female & 6 & 85 & & 6 & 3 & \\
\hline $\mathrm{BMI}, \mathrm{kg} / \mathrm{m}^{2}$ & $22.77 \pm 3.29$ & $23.06 \pm 9.58$ & 0.680 & $22.77 \pm 3.29$ & $22.67 \pm 3.55$ & 0.920 \\
\hline Presence of comorbidity, $n$ & & & 0.694 & & & 0.771 \\
\hline Yes & 10 & 219 & & 10 & 9 & \\
\hline No & 15 & 387 & & 15 & 16 & \\
\hline AFP, $n$ & & & 0.171 & & & 0.777 \\
\hline$\leq 400 \mu \mathrm{g} / 1$ & 14 & 188 & & 14 & 13 & \\
\hline$>400 \mu \mathrm{g} / 1$ & 11 & 418 & & 11 & 12 & \\
\hline $\mathrm{HBV}, \mathrm{n}$ & & & 0.212 & & & $0.480^{\mathrm{a}}$ \\
\hline Positive & 19 & 516 & & 19 & 21 & \\
\hline Negative & 6 & 90 & & 6 & 4 & \\
\hline $\mathrm{HCV}, \mathrm{n}$ & & & $1.000^{\mathrm{b}}$ & & & $1.000^{\mathrm{b}}$ \\
\hline Positive & 0 & 17 & & 0 & 0 & \\
\hline Negative & 25 & 589 & & 25 & 25 & \\
\hline ICGR $15, \%$ & $7.53 \pm 4.36$ & $6.96 \pm 3.87$ & 0.396 & $7.53 \pm 4.36$ & $7.25 \pm 3.66$ & 0.810 \\
\hline Child-Pugh classification $^{\mathrm{c}}, \mathrm{n}$ & & & $0.002^{\mathrm{a}}$ & & & $0.221^{\mathrm{a}}$ \\
\hline $\mathrm{A}$ & 20 & 586 & & 20 & 23 & \\
\hline $\mathrm{B}$ & 5 & 20 & & 5 & 2 & \\
\hline Maximum tumour size, $\mathrm{cm}$ & $6.97 \pm 3.45$ & $5.15 \pm 3.58$ & 0.019 & $6.97 \pm 3.45$ & $6.51 \pm 5.12$ & 0.714 \\
\hline Number of tumours, $n$ & & & $0.461^{\mathrm{a}}$ & & & $1.000^{\mathrm{a}}$ \\
\hline Solitary & 21 & 538 & & 21 & 21 & \\
\hline Multiple & 4 & 68 & & 4 & 4 & \\
\hline Cirrhosis, $\mathrm{n}$ & & & 0.001 & & & 0.145 \\
\hline Yes & 7 & 366 & & 7 & 12 & \\
\hline No & 18 & 240 & & 18 & 13 & \\
\hline Macrovascular invasion, $\mathrm{n}$ & & & $0.002^{\mathrm{a}}$ & & & $0.440^{\mathrm{a}}$ \\
\hline Yes & 5 & 31 & & 5 & 3 & \\
\hline No & 20 & 575 & & 20 & 22 & \\
\hline Microvascular invasion, $\mathrm{n}$ & & & $<0.001$ & & & 0.089 \\
\hline Yes & 15 & 153 & & 15 & 9 & \\
\hline No & 10 & 453 & & 10 & 16 & \\
\hline Tumour differentiation, $\mathrm{n}$ & & & $0.003^{\mathrm{a}}$ & & & $0.172^{\mathrm{a}}$ \\
\hline Well & 0 & 36 & & 0 & 2 & \\
\hline Moderate & 12 & 429 & & 12 & 15 & \\
\hline Poor & 13 & 141 & & 13 & 8 & \\
\hline AJCC stage $^{\mathrm{c}}, \mathrm{n}$ & & & 0.001 & & & $0.505^{\mathrm{a}}$ \\
\hline I & 8 & 403 & & 8 & 12 & \\
\hline II & 11 & 148 & & 11 & 8 & \\
\hline III & 6 & 55 & & 6 & 5 & \\
\hline Resection margins, $\mathrm{n}$ & & & $0.026^{\mathrm{a}}$ & & & $0.157^{\mathrm{a}}$ \\
\hline R0 & 21 & 592 & & 21 & 24 & \\
\hline $\mathrm{R} 1$ & 4 & 14 & & 4 & 1 & \\
\hline
\end{tabular}

Continuous variables are presented as the mean \pm SD. ${ }^{a}$ Fisher's exact test. ${ }^{\mathrm{b}}$ Child-Pugh classification system: Class A, 5-6 points; class B, 7-9 points. ${ }^{\mathrm{c}} \mathrm{Stage} \mathrm{Ia}$ or Ib and IIIa or IIIb were classified together as stage I and III, respectively. Tumour stage was classified in accordance with the criteria of the AJCC 8th edition. AFP, $\alpha$-fetoprotein; BMI, body mass index; HBV, hepatitis B virus; HCV, hepatitis C virus; ICGR15, indocyanine green retention rate at $15 \mathrm{~min}$; AJCC, American Joint Committee on Cancer; BDTT, bile duct tumour thrombus. 

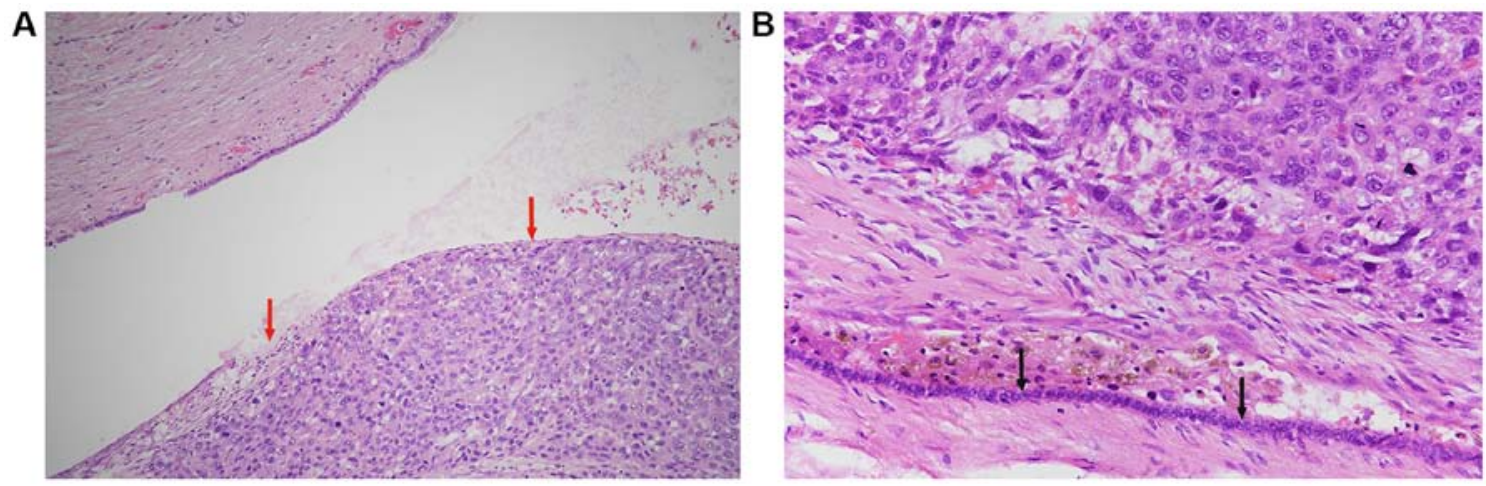

Figure 1. Histopathological findings of BDTT analyzed via hematoxylin and eosin staining. (A) BDTT (red arrow) did not adhere to the bile duct wall around it (magnification, x100). (B) The BDTT and bile duct wall are separated by fibrous tissue, and pro-fibrotic reactions are easily formed between the BDTT and bile duct epithelium (black arrow) (magnification, x400). BDTT, bile duct tumour thrombus.

A

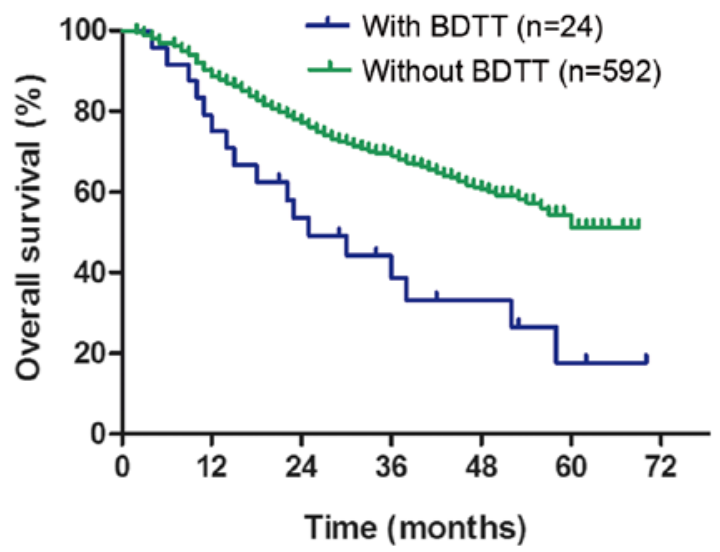

C

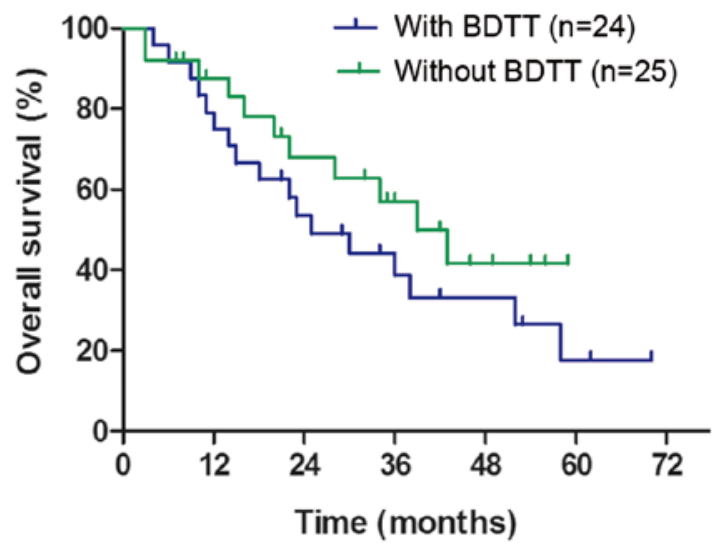

B
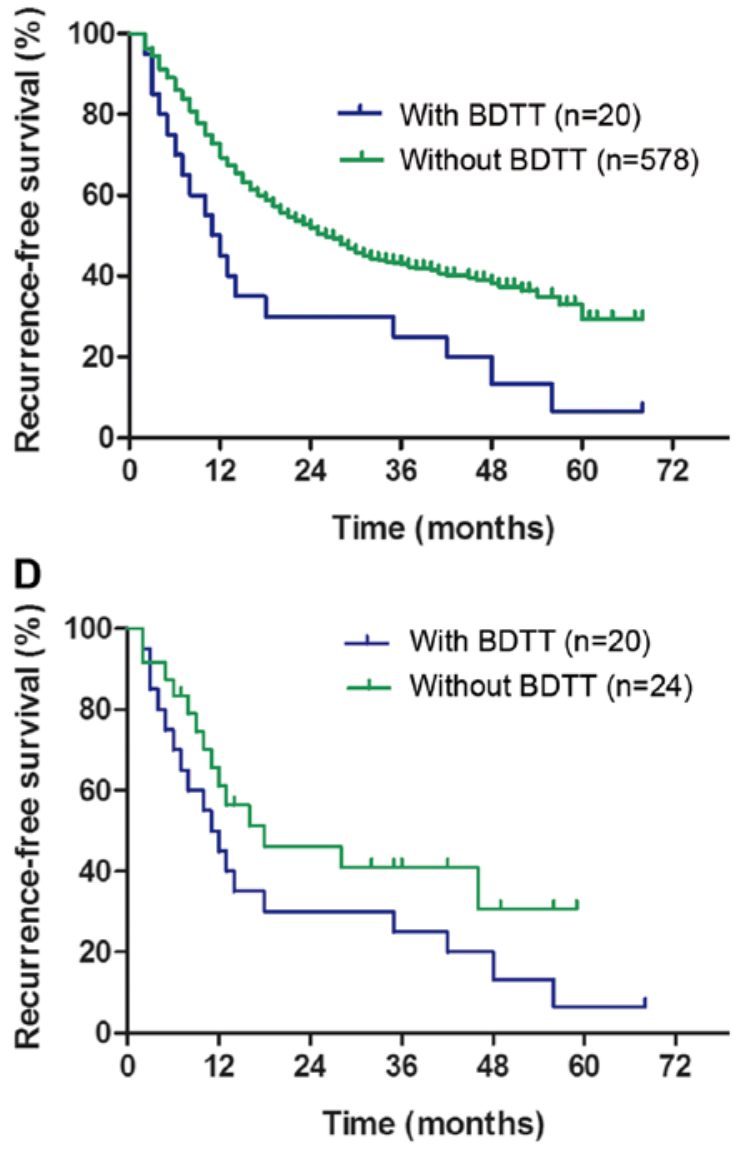

Figure 2. OS and RFS curves between two groups before and after PSM. (A) The OS of the BDTT group was significantly poorer than that of group without BDTT before PSM. (B) The RFS of the BDTT group was significantly poorer than that of the group without BDTT before PSM. (C) The OS comparison between the groups with and without BDTT revealed no significant difference after PSM. (D) The RFS comparison between the groups with and without BDTT revealed no significant difference after PSM. OS, overall survival; RFS, recurrence-free survival; PSM, propensity score matching; BDTT, bile duct tumour thrombus.

$(12 \%)$ and by right posterior sectionectomy in 2 patients $(8 \%)$ Caudate lobectomy was performed in 8 patients (32\%). The BDTT was removed by thrombectomy either through choledochotomy or the cut end of the bile duct in 11 patients (44\%), and extrahepatic bile duct resection was performed in 4 patients (16\%). In the remaining 10 patients (40\%), BDTTs were resectioned en bloc together with the primary tumour. Compared with those in the group without BDTT, the average operative time and intraoperative blood loss in the BDTT group were significantly greater $(249.67 \pm 80.54$ vs. $166.75 \pm 55.73 \mathrm{~min}$, $\mathrm{P}<0.001$; and $966.67 \pm 917.16$ vs. $352.86 \pm 335.72 \mathrm{ml}, \mathrm{P}=0.012$, respectively). The perioperative mortality rate was not significantly different between the two groups (4.0 vs. $1.16 \%$; $\mathrm{P}=0.287$ ) (data not shown). 

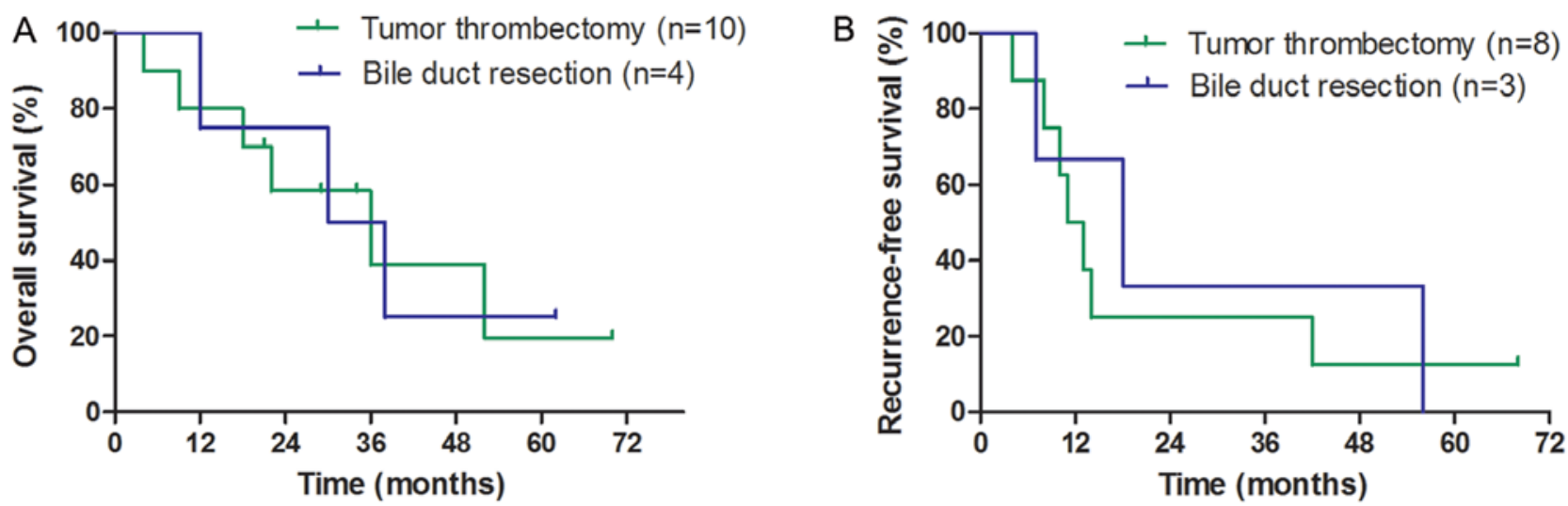

Figure 3. Postoperative OS and RFS curves for HCC patients with Ueda type 3 bile duct tumour thrombus according to bile duct resection or absence of bile duct resection. (A) OS comparison between HCC patients who underwent bile duct resection and tumour thrombectomy revealed no significant difference. (B) RFS comparison between HCC patients who underwent bile duct resection and tumour thrombectomy revealed no significant difference. OS, overall survival; RFS, recurrence-free survival; HCC, hepatocellular carcinoma.

Clinicopathological characteristics before and after PSM. The clinicopathological characteristics of the two groups before and after PSM are shown in Table II. The proportion of females and AFP levels in the BDTT group seemed to be higher, but these results did not reach statistical significance. Fewer patients had a Child-Pugh A classification in the BDTT group than in the group without BDTT just before surgery $(\mathrm{P}=0.002)$, which corresponds to the aforementioned result that the preoperative TBIL level in the BDTT group was significantly higher than that in the group without BDTT. The numbers of tumours were not significantly different between the two groups, while the tumour diameter in the BDTT group was significantly larger than that in the group without BDTT $(\mathrm{P}=0.019)$. In the BDTT group, 5 patients $(20 \%)$ had macrovascular invasion and 15 patients $(60 \%)$ had microvascular invasion, with both values being significantly higher than those in the group without BDTT $(\mathrm{P}=0.002$ and $\mathrm{P}<0.001$, respectively). The Edmondson-Steiner grade and AJCC stage in the BDTT group were also significantly higher than those in the group without BDTT ( $\mathrm{P}=0.003$ and $\mathrm{P}=0.001$, respectively). However, the presence of cirrhosis was significantly lower in the BDTT group than in the group without BDTT $(\mathrm{P}=0.001)$.

The imbalance in clinicopathological characteristics between the two groups indicates that the data between the two groups were incomparable before PSM. To balance this difference, the clinicopathological indices were set as matching covariates for PSM. The aforementioned variables were not significantly different between these two groups after PSM $(\mathrm{P}>0.05)$, indicating that the two sets of data were comparable (Table II).

Postoperative pathological features. Although the most common location of BDTT was the CBD (60\%) in the present study, BDTT rarely invaded the wall of the CBD histologically. The underlying epithelium of the resected large bile ducts was well preserved based on the histological examination of the 4 patients who underwent extrahepatic bile duct resection in this study. One of the postoperative histopathological findings was that BDTT did not usually adhere to the CBD. The cancer cells in BDTT were flaky and solid, the cells had obvious atypia and pleomorphism, and pathological mitosis was not uncommon (Fig. 1A). Although the tumour cells were scattered and haemosiderin deposition was evident, suggesting old bleeding, biliary epithelial cells were still continuous and intact, and were protected by profibrotic reactions (Fig. 1B).

Postoperative long-term outcomes. Patients who died perioperatively were excluded from the survival analysis. The median follow-up duration was 32 months (range, 1-70 months). The cumulative 1-, 3- and 5-year overall survival (OS) rates in the group without BDTT $(88.61,69.01$ and $51.16 \%$, respectively) were significantly higher than those in the BDTT group (75.00, 38.67 and $17.68 \%$, respectively) $(\mathrm{P}<0.001$; Fig. 2A). The cumulative 1-, 3- and 5-year recurrence-free survival (RFS) rates in the group without BDTT (69.32, 43.01 and $29.40 \%$, respectively) were significantly higher than those in the BDTT group $(45.00,25.00$, and $6.67 \%$, respectively) $(\mathrm{P}=0.003$; Fig. 2B). However, no significant differences in the cumulative 1-, 3- and 5-year OS or RFS rates were identified between the two groups after PSM (OS: 87.62, 57.07 and 41.61\%, respectively, vs. 75.00, 38.67 and $17.68 \%$, respectively; $\mathrm{P}=0.249$; Fig. 2C; RFS: $61.09,41.01$ and $30.76 \%$, respectively, vs. 45.00 , 25.00 and $6.67 \%$, respectively; $\mathrm{P}=0.121$; Fig. $2 \mathrm{D}$ ). These results illustrate that covariates other than BDTT also affect the RFS and OS rates.

In the BDTT group, the median OS and RFS times of patients who underwent tumour thrombectomy and bile duct resection were not significantly different (OS: 36 vs. 30 months, respectively; $\mathrm{P}=0.891$; Fig. $3 \mathrm{~A}$; RFS: 11 vs. 18 months, respectively; $\mathrm{P}=0.787$; Fig. 3B). A total of $17 \mathrm{HCC}$ (68.0\%) patients with BDTT experienced recurrence during the follow-up; intrahepatic recurrence was the most common type [12/17 (70.59\%)], followed by combined intrahepatic and extrahepatic recurrence [3/17 (17.65\%)] and extrahepatic recurrence [2/17 (11.76\%)], both of which were in the lungs (data not shown). None of the patients who underwent tumour thrombectomy exhibited peritoneal dissemination, and only one of these patients experienced bile duct recurrence and underwent re-resection.

Univariate and multivariate analyses of OS. Univariate and multivariate analyses were performed by Cox proportional 
Table III. Univariate and multivariate cox proportional analysis for overall survival.

\begin{tabular}{|c|c|c|c|c|c|c|c|}
\hline \multirow[b]{2}{*}{ Variables } & \multirow[b]{2}{*}{$\mathrm{n}$} & \multicolumn{3}{|c|}{ Univariate analysis } & \multicolumn{3}{|c|}{ Multivariate analysis } \\
\hline & & HR & $95 \% \mathrm{CI}$ & P-value & HR & $95 \% \mathrm{CI}$ & P-value \\
\hline \multicolumn{8}{|l|}{ Group } \\
\hline Without BDTT & 25 & 1.000 & & & & & \\
\hline With BDTT & 24 & 1.558 & $0.727-3.341$ & 0.254 & & & \\
\hline \multicolumn{8}{|l|}{ Age, years } \\
\hline$<60$ & 31 & 1.000 & & & & & \\
\hline$\geq 60$ & 18 & 1.230 & $0.5745-2.633$ & 0.593 & & & \\
\hline \multicolumn{8}{|l|}{ Gender } \\
\hline Male & 40 & 1.000 & & & & & \\
\hline Female & 9 & 1.242 & $0.464-3.323$ & 0.666 & & & \\
\hline \multicolumn{8}{|c|}{ Presence of comorbidity } \\
\hline No & 31 & 1.000 & & & & & \\
\hline Yes & 18 & 1.151 & $0.543-2.441$ & 0.715 & & & \\
\hline \multicolumn{8}{|l|}{$\mathrm{AFP}, \mu \mathrm{g} / 1$} \\
\hline$<400$ & 26 & 1.000 & & & & & \\
\hline$\geq 400$ & 23 & 1.617 & $0.764-3.423$ & 0.209 & & & \\
\hline \multicolumn{8}{|c|}{$\mathrm{HBV}$ virus antigen } \\
\hline Negative & 9 & 1.000 & & & & & \\
\hline Positive & 40 & 1.005 & $0.405-2.498$ & 0.991 & & & \\
\hline \multicolumn{8}{|l|}{ ICGR15a,$\%$} \\
\hline$\leq 10$ & 22 & 1.000 & & & & & \\
\hline$>10$ & 8 & 2.417 & $0.703-8.307$ & 0.161 & & & \\
\hline \multicolumn{8}{|c|}{ Child-Pugh classification } \\
\hline A & 42 & 1.000 & & & & & \\
\hline B & 7 & 1.655 & $0.666-4.113$ & 0.278 & & & \\
\hline \multicolumn{8}{|c|}{ Maximum tumour size, $\mathrm{cm}$} \\
\hline$>5$ & 24 & 1.000 & & & & & \\
\hline$\geq 5$ & 25 & 1.614 & 0.743-3.506 & 0.226 & & & \\
\hline \multicolumn{8}{|c|}{ Number of tumours } \\
\hline Solitary & 41 & 1.000 & & & & & \\
\hline Multiple & 8 & 1.717 & $0.725-4.065$ & 0.219 & & & \\
\hline \multicolumn{8}{|l|}{ Cirrhosis } \\
\hline No & 31 & 1.000 & & & & & \\
\hline Yes & 18 & 1.27 & $0.587-2.747$ & 0.544 & & & \\
\hline \multicolumn{8}{|c|}{ Macrovascular invasion } \\
\hline Negative & 41 & 1.000 & & & 1.000 & & \\
\hline Positive & 8 & 4.613 & $1.944-10.947$ & 0.001 & 3.220 & $1.184-8.756$ & 0.022 \\
\hline \multicolumn{8}{|c|}{ Microvascular invasion } \\
\hline Negative & 24 & 1.000 & & & 1.000 & & \\
\hline Positive & 25 & 2.942 & $1.350-6.413$ & 0.007 & 1.728 & $0.737-4.051$ & 0.208 \\
\hline \multicolumn{8}{|c|}{ Tumour differentiation } \\
\hline Well $^{\mathrm{b}}$ & 2 & - & - & - & - & - & - \\
\hline Moderate & 27 & 1.000 & & & 1.000 & & \\
\hline Poor & 20 & 2.302 & $1.081-4.905$ & 0.031 & 2.150 & $0.968-4.775$ & 0.06 \\
\hline \multicolumn{8}{|l|}{ Resection margins } \\
\hline R0 & 44 & 1.000 & & & 1.000 & & \\
\hline R1 & 5 & 5.715 & $2.065-15.819$ & 0.001 & 2.563 & $0.789-8.326$ & 0.117 \\
\hline
\end{tabular}

${ }^{\mathrm{a}}$ In the early years, some patients did not undergo the ICG15 test so $\mathrm{n}=30$ only. ${ }^{\mathrm{b}}$ Due to the small number of cases in these groups, the survival analysis was meaningless. HR, hazard ratio; CI, confidence interval; AFP, $\alpha$-fetoprotein; HBV, hepatitis B virus; ICGR15, indocyanine green retention rate at $15 \mathrm{~min}$; BDTT, bile duct tumour thrombus. 
hazards regression in 49 patients who underwent PSM. The univariate analysis revealed that macrovascular invasion (HR, 4.613; $\mathrm{P}=0.001)$, microvascular invasion $(\mathrm{HR}, 2.942 ; \mathrm{P}=0.007)$, tumour differentiation $(\mathrm{HR}, 2.302 ; \mathrm{P}=0.031)$ and resection margins (HR, 5.715; $\mathrm{P}=0.001)$, rather than BDTT (HR, 1.558; $\mathrm{P}=0.254$ ), significantly affected OS (Table III). These potential risk factors were further examined by multivariate Cox proportional hazards regression analysis. The results revealed that macrovascular invasion (HR, 3.220; 95\% CI, 1.184-8.756; $\mathrm{P}=0.022$ ) was the only independent predictor of OS (Table III).

\section{Discussion}

Manifestations of jaundice in patients with HCC are thought to be caused by massive tumour infiltration of the liver parenchyma or advanced underlying liver cirrhosis $(16,17)$. Sometimes, precisely distinguishing the cause of jaundice is difficult in clinical practice (18); therefore, patients with BDTT and jaundice are often forced to receive conservative treatment, thus missing the opportunity for suitable treatment with a potential chance of a complete response. This is mainly due to insufficient understanding of the clinicopathological characteristics of the tumour. Although a general consensus indicates that the oncological prognosis of surgical treatment is significantly improved compared with that of palliative treatment $(11,12)$, studies on the surgical treatment of HCC with BDTT are uncommon, especially studies regarding postoperative long-term outcomes. Whether the postoperative survival of patients with HCC and BDTT is equal to that of patients with HCC and no BDTT $(2,8,18-24)$ and whether the extrahepatic bile duct should be removed remain controversial $(9,25,26)$.

As reported in previous studies $(24,27,28)$, compared with HCC without BDTT, HCC with BDTT demonstrates clinicopathological features such as a longer tumour diameter, a higher incidence of vascular invasion and a higher Edmondson-Steiner grade, AJCC stage and Child-Pugh classification; however, cirrhosis is less common (28), which may be associated with the biological behaviour of HCC with BDTT. The current results (Table II) before PSM are consistent with these earlier findings, which decreases the comparability of these studies. PSM could have balanced the confounding variables and reduced the impact of selection biases, and the results would have been similar to those of randomized controlled studies $(29,30)$. The clinicopathological features between the two groups after PSM were not significantly different, which increased the comparability between groups. Therefore, PSM was used to control for selection bias in the current study, increasing the credibility of the research results.

Another notable area for comparison is whether postoperative survival among $\mathrm{HCC}$ patients with BDTT is equal to that among HCC patients without BDTT. Yang et al (21) reported that the median OS time in the BDTT group (16.6 months) was significantly worse than that in the group without BDTT (84.0 months). Other studies $(8,22,23)$ have also illustrated that, compared with the OS in the group without BDTT, the OS of the BDTT group was significantly poorer. However, Shiomi et al (16) reported that the 3- and 5-year OS rates (47 and $28 \%$, respectively) in the BDTT group were not significantly different from those in the group without BDTT
(63 and 48\%, respectively; $\mathrm{P}=0.190$ ). The reason for this difference may be that the potential impact of other variables on the postoperative survival of patients with HCC and BDTT was neglected $(2,24)$. In the present study, the 3- and 5-year OS rates in the BDTT group were worse than those in the group without BDTT before PSM. However, no significant difference was observed between the two groups after PSM. These results show that when matching other variables and balancing selection bias, BDTT does not affect the long-term survival of patients with $\mathrm{HCC}$ who receive liver resection.

The RFS rates in the BDTT group were significantly worse than those in the group without BDTT before PSM in the present study, which is consistent with the results of previous studies $(8,21)$. However, Kim et al (22) reported that RFS rates between the two groups were not significantly different. Similarly, in the current study, no significant differences in RFS rates were found between the two groups after PSM. These results indicate that BDTT does not affect postoperative RFS in patients with HCC. Tumour recurrence is generally accepted to affect survival in patients with HCC who undergo surgery with curative intent. Zeng et al (7) also reported that tumour recurrence was an unfavourable prognostic factor for OS. BDTT did not affect postoperative RFS in patients with $\mathrm{HCC}$, which indirectly illustrates that it is not an unfavourable prognostic factor for OS after surgery. All the results indicate that BDTT in HCC has low indolent malignant potential and is not a contraindication for liver resection.

HCC with portal vein tumour thrombus (PVTT) is recognized as a relatively advanced-stage disease regardless of the criteria of the Barcelona Clinic for Liver Cancer Staging System (31) or the AJCC 8th Edition. HCC patients with PVTT have a highly unfavourable prognosis with regard to macrovascular invasion, often causing the widespread dissemination of cancer cells and leading to intrahepatic metastasis, which is considered an important mechanism of intrahepatic recurrence $(32,33)$. In the present study, the results of the multivariate analysis revealed that macrovascular invasion was one of the independent prognostic risk factors for the long-term survival of HCC patients after resection, which is similar to the results of previous studies (28). These findings may be associated with the highly aggressive biological behaviour of HCC with macrovascular invasion. Non-curative liver resection was deemed another independent unfavourable risk factor for postoperative long-term survival in HCC patients in the univariate analysis, but not in the multivariate analysis, in the present study. The reason for this finding may be that the number of patients who underwent non-curative resection was insufficient, and its statistical weight was inadequate. Therefore, we hypothesize that when the number of non-curative surgical resections increases, non-curative resection may increase the risk of impaired long-term postoperative survival, which is a theory that is widely accepted by some surgeons $(34,35)$. Therefore, if associated conditions are permitted, curative resection is recommended when the liver function of these patients is sufficient after appropriate preoperative management.

Whether extrahepatic bile duct resection for $\mathrm{HCC}$ with BDTT is necessary remains controversial. Moon et al (25) claimed that if BDTT could be successfully removed, bile duct resection may not be necessary. Moreover, Noda et al (28) 
and Shibata et al (36) illustrated that the potential for serious complications, such as liver abscesses, may not be avoided when intrahepatic recurrence is treated locally after bile duct resection. Neither the median survival time nor the RFS time between the bile duct resection and tumour thrombectomy groups was significantly different in the present study, which is consistent with the results of previous studies $(16,19)$. The surgical treatment strategy in the current study was the complete resection of primary HCC and BDTT, and it aimed not to remove the extrahepatic bile duct; this is similar to the 'peeling off technique' (10). Tumour thrombectomy is believed to have potential risks of peritoneal dissemination and recurrence in the preserved bile duct. However, Kim et al (37) proclaimed that thrombectomy does not significantly increase the risk of peritoneal metastasis. None of the patients who underwent tumour thrombectomy presented with peritoneal dissemination in the present study. Although bile duct recurrence was observed in one of these patients, the risks are considered minimal as the first recurrence most frequently occurs in the remnant liver and not in the bile duct, and the opportunity to undergo curative re-resection is still available.

The absence of bile duct resection for patients with HCC and BDTT may cause recurrence due to direct invasion of the tumour into the bile duct, periductal capillary plexus and lymphatic system $(27,38)$. Recently, a multicentre study reported that bile duct resection was a significant favourable prognostic factor for recurrence, OS and survival after recurrence (37). Based on the aforementioned studies, the selection criteria for resecting the bile duct could not be too absolute, and an individualized treatment plan was required according to the condition of each patient. Whether extrahepatic bile duct resection is necessary for patients with HCC with BDTT should be further explored. Regardless of the surgical approach to remove or retain the bile duct, curative resection of the tumour is the most important aim.

The present study also has some limitations. As a retrospective case-control study, although a control group was established for direct comparisons, the number of patients with BDTT was insufficient. The reasons may be as follows: i) The incidence of HCC with BDTT is rare (2-6); ii) the biological and clinicopathological characteristics of the tumours were inadequately understood, therefore, many patients did not receive surgical treatment in the early period; and iii) the clinicopathological data of these patients were incomplete in the early period and were thus excluded from the study. Considering the rarity of HCC with BDTT, the present study is of great clinical significance, as the number of included patients exceeds that in numerous other studies $(2,10,16,28,34)$. Although PSM was used to reduce the imbalance caused by selection bias, some unavoidable confounding variables remain. For instance, the preoperative TBIL level in the BDTT group was significantly higher than that in the group without BDTT before and after PSM. However, the impact of the preoperative TBIL level on survival was reflected in the Child-Pugh classification system, and it was not identified as an important prognostic factor for patients with BDTT. Large, multicentre, prospective, randomized, controlled trials are still needed for further validation.

Compared with those of patients without BDTT, the clinicopathological features of patients with HCC and BDTT are more advanced, and the long-term postoperative outcome of these patients is worse. Macrovascular invasion, but not BDTT, is a significantly unfavourable risk factor for the survival of patients with HCC who undergo liver resection. Curative resection is recommended for patients with HCC and BDTT, even for those with poor liver function after proper perioperative management, in order to achieve good long-term survival.

\section{Acknowledgements}

Not applicable.

\section{Funding}

This study was funded by Construction Projects of State Key Clinical Specialty of Health Department (grant no. 2013-GJLCZD).

\section{Availability of data and materials}

The datasets used and/or analyzed during the present study are available from the corresponding author on reasonable request.

\section{Authors' contributions}

QC analyzed and interpreted the patient data, and was a major contributor in writing the manuscript. SW was involved in study design, study supervision and critical revision of the manuscript. ZS, ZZ and $\mathrm{XZ}$ analyzed and interpreted the patient data. LZ performed the histological examination of the HCC samples. All authors read and approved the final manuscript.

\section{Ethics approval and consent to participate}

Written informed consent was obtained from all individuals included in the study at the time of initial data collection. The study was approved by the Institutional Review Board of The First Affiliated Hospital of Fujian Medical University and performed in accordance with the ethical guidelines of the institute.

\section{Patient consent for publication}

Not applicable.

\section{Competing interests}

The authors declare that they have no competing interests.

\section{References}

1. Shehta A, Han HS, Yoon YS, Cho JY and Choi Y: Laparoscopic liver resection for hepatocellular carcinoma in cirrhotic patients: 10-year single-center experience. Surg Endosc 30: 638-648, 2016.

2. Oba A, Takahashi S, Kato Y, Gotohda N, Kinoshita T, Shibasaki H, Ikeda M and Konishi M: Usefulness of resection for hepatocellular carcinoma with macroscopic bile duct tumor thrombus. Anticancer Res 34: 4367-4372, 2014.

3. Ueda M, Takeuchi T, Takayasu T, Takahashi K, Okamoto S, Tanaka A, Morimoto T, Mori K and Yamaoka Y: Classification and surgical treatment of hepatocellular carcinoma (HCC) with bile duct thrombi. Hepatogastroenterology 41: 349-354, 1994. 
4. Kudo M, Izumi N, Ichida T, Ku Y, Kokudo N, Sakamoto M, Takayama T, Nakashima O, Matsui O and Matsuyama Y: Report of the 19th follow-up survey of primary liver cancer in Japan. Hepatol Res 46: 372-390, 2016.

5. Nakashima T, Okuda K, Kojiro M, Jimi A, Yamaguchi R, Sakamoto K and Ikari T: Pathology of hepatocellular carcinoma in Japan. 232 consecutive cases autopsied in ten years. Cancer 51: 863-877, 1983.

6. Meng KW, Dong M, Zhang WG and Huang QX: Clinical characteristics and surgical prognosis of hepatocellular carcinoma with bile duct invasion. Gastroenterol Res Pract 2014: 604971, 2014

7. Zeng H, Xu LB, Wen JM, Zhang R, Zhu MS, Shi XD and Liu C: Hepatocellular carcinoma with bile duct tumor thrombus: A clinicopathological analysis of factors predictive of recurrence and outcome after surgery. Medicine (Baltimore) 94: e364, 2015

8. Shao W, Sui C, Liu Z, Yang J and Zhou Y: Surgical outcome of hepatocellular carcinoma patients with biliary tumor thrombi World J Surg Oncol 9: 2, 2011.

9. Ikenaga N, Chijiiwa K, Otani K, Ohuchida J, Uchiyama S and Kondo K: Clinicopathologic characteristics of hepatocellular carcinoma with bile duct invasion. J Gastrointest Surg 13: 492-497, 2009.

10. Yamamoto S, Hasegawa K, Inoue Y, Shindoh J, Aoki T, Sakamoto Y, Sugawara Y, Makuuchi M and Kokudo N: Bile duct preserving surgery for hepatocellular carcinoma with bile duct tumor thrombus. Ann Surg 261: e123-e125, 2015.

11. Xiangji L, Weifeng T, Bin Y, Chen L, Xiaoqing J, Baihe Z, Feng S and Mengchao W: Surgery of hepatocellular carcinoma complicated with cancer thrombi in bile duct: Efficacy for criteria for different therapy modalities. Langenbecks Arch Surg 394: 1033-1039, 2009.

12. Lau W, Leung K, Leung TW, Liew CT, Chan MS, Yu SC and Li AK: A logical approach to hepatocellular carcinoma presenting with jaundice. Ann Surg 225: 281-285, 1997.

13. Bae SH, Park HC, Yoon WS, Yoon SM, Jung IH, Lee IJ, Kim JW Seong J, Kim TH, Nam TK, et al: Treatment outcome after fractionated conformal radiotherapy for hepatocellular carcinoma in patients with child-pugh classification B in Korea (KROG 16-05). Cancer Res Treat 51: 1589-1599, 2019.

14. Abdel-Rahman O: Assessment of the discriminating value of the 8th AJCC stage grouping for hepatocellular carcinoma. HPB (Oxford) 20: 41-48, 2018.

15. Edmondson HA and Steiner PE: Primary carcinoma of the liver: A study of 100 cases among 48,900 necropsies. Cancer 7: 462-503, 1954

16. Shiomi M, Kamiya J, Nagino M, Uesaka K, Sano T, Hayakawa N, Kanai M, Yamamoto H and Nimura Y: Hepatocellular carcinoma with biliary tumor thrombi: Aggressive operative approach after appropriate preoperative management. Surgery 129: 692-698, 2001.

17. Lee NW, Wong KP, Siu KF and Wong J: Cholangiography in hepatocellular carcinoma with obstructive jaundice. Clin Radiol 35: 119-123, 1984.

18. Mok KT, Chang HT, Liu SI, Jou NW, Tsai CC and Wang BW: Surgical treatment of hepatocellular carcinoma with biliary tumor thrombi. Int Surg 81: 284-288, 1996.

19. Satoh S, Ikai I, Honda G, Okabe H, Takeyama O, Yamamoto Y Yamamoto N, Iimuro Y, Shimahara Y and Yamaoka Y: Clinicopathologic evaluation of hepatocellular carcinoma with bile duct thrombi. Surgery 128: 779-783, 2000.

20. Yeh CN, Jan YY, Lee WC and Chen MF: Hepatic resection for hepatocellular carcinoma with obstructive jaundice due to biliary tumor thrombi. World J Surg 28: 471-475, 2004.

21. Yang X, Qiu Z, Ran R, Cui L, Luo X, Wu M, Tan WF and Jiang X: Prognostic importance of bile duct invasion in surgical resection with curative intent for hepatocellular carcinoma using PSM analysis. Oncol Lett 16: 3593-3602, 2018.

22. Kim JM, Kwon CH, Joh JW, Sinn DH, Park JB, Lee JH, Kim SJ, Paik SW, Park CK and Yoo BC: Incidental microscopic bile duct tumor thrombi in hepatocellular carcinoma after curative hepatectomy: A matched study. Medicine (Baltimore) 94: e450, 2015
23. Rammohan A, Sathyanesan J, Rajendran K, Pitchaimuthu A Perumal SK, Balaraman K, Ramasamy R, Palaniappan R and Govindan M: Bile duct thrombi in hepatocellular carcinoma: Is aggressive surgery worthwhile? HPB (Oxford) 17: 508-513, 2015.

24. Wong TC, Cheung TT, Chok KS, Chan AC, Dai WC, Chan SC, Poon RT, Fan ST and Lo CM: Outcomes of hepatectomy for hepatocellular carcinoma with bile duct tumour thrombus. HPB (Oxford) 17: 401-408, 2015.

25. Moon DB, Hwang S, Wang HJ, Yun SS, Kim KS, Lee YJ, Kim KH, Park YK, Xu W, Kim BW, et al: Surgical outcomes of hepatocellular carcinoma with bile duct tumor thrombus: A Korean multicenter study. World J Surg 37: 443-451, 2013.

26. Yasunori $\mathrm{M}$ and Masatoshi K: Hepatocellular carcinoma with obstructive jaundice: Endoscopic and percutaneous biliary drainage. Dig Dis 30: 592-597, 2012.

27. Orimo T, Kamiyama T, Yokoo H, Wakayama K, Shimada S, Tsuruga Y, Kamachi H and Taketomi A: Hepatectomy for hepatocellular carcinoma with bile duct tumor thrombus, including cases with obstructive Jaundice. Ann Surg Oncol 23: 2627-2634, 2016.

28. Noda T, Nagano H, Tomimaru Y, Murakami M, Wada H, Kobayashi S, Marubashi S, Eguchi H, Takeda Y, Tanemura M, et al: Prognosis of hepatocellular carcinoma with biliary tumor thrombi after liver surgery. Surgery 149: 371-377, 2011.

29. Rubin DB: Estimating causal effects from large data sets using propensity scores. Ann Intern Med 127: 757-263, 1997.

30. Austin PC: An introduction to propensity score methods for reducing the effects of confounding in observational studies. Multivariate Behav Res 46: 399-424, 2011.

31. Cho HJ, Kim SS, Kang SY, Yang MJ, Noh CK, Hwang JC, Lim SG, Shin SJ, Lee KM, Yoo BM, et al: A proposal for modification of the barcelona clinic liver cancer staging system considering the prognostic implication of performance status. Gut Liver 13: 557-568, 2019.

32. Vauthey JN, Klimstra D, Franceschi D, Tao Y, Fortner J, Blumgart L and Brennan M: Factors affecting long-term outcome after hepatic resection for hepatocellular carcinoma. Am J Surg 169: 28-35, 1995.

33. Yamamoto J, Kosuge T, Takayama T, Shimada K, Yamasaki S, Ozaki H, Yamaguchi N and Makuuchi M: Recurrence of hepatocellular carcinoma after surgery. Br J Surg 83: 1219-1222, 1996.

34. Esaki M, Shimada K, Sano T, Sakamoto Y, Kosuge T and Ojima H: Surgical results for hepatocellular carcinoma with bile duct invasion: A clinicopathologic comparison between macroscopic and microscopic tumor thrombus. J Surg Oncol 90: 226-232, 2005.

35. Han J, Li ZL, Xing H, Wu H, Zhu P, Lau WY, Zhou YH, Gu WM, Wang $\mathrm{H}$, Chen TH, et al: The impact of resection margin and microvascular invasion on long-term prognosis after curative resection of hepatocellular carcinoma: A multi-institutional study. HPB (Oxford) 21: 962-971, 2019.

36. Shibata T, Yamamoto N, Ikai I, Shimahara Y, Yamaoka Y, Itoh K and Konishi J: Choledochojejunostomy: Possible risk factor for septic complications after percutaneous hepatic tumor ablation. AJR Am J Roentgenol 174: 985-986, 2000.

37. Kim DS, Kim BW, Hatano E, Hwang S, Hasegawa K, Kudo A, Ariizumi S, Kaibori M, Fukumoto T, Baba H, et al: Surgical outcomes of hepatocellular carcinoma with bile duct tumor thrombus: A Korea-Japan multicenter study. Ann Surg 271: 913-921, 2020.

38. Kojiro M, Kawabata K, Kawano Y, Shirai F, Takemoto N and Nakashima T: Hepatocellular carcinoma presenting as intrabile duct tumor growth: A clinicopathologic study of 24 cases. Cancer 49: 2144-2147, 1982.

This work is licensed under a Creative Commons Attribution-NonCommercial-NoDerivatives 4.0 International (CC BY-NC-ND 4.0) License. 